\title{
Superiority of QTL-Assisted Selection in Dairy Cattle Breeding Schemes
}

\author{
G. Abdel-Azim and A. E. Freeman \\ Department of Animal Science, \\ lowa State University, Ames 50011
}

\section{ABSTRACT}

The superiority of selection schemes employing information about a known quantitative trait locus (QTL) over conventional schemes is examined for dairy cattle breeding schemes. Stochastic simulation of a dairy cattle population with selection practices, structures, and parameters similar to the US Holstein population was implemented. Additive genetic effects were estimated by an animal model. Two schemes were compared: a QTL-assisted selection scheme in which the genotype of a known QTL was accounted for in the animal model as a fixed factor, and a QTL-free selection scheme in which the QTL was simulated but was not fit separately in the animal model. Under the QTL-assisted selection scheme, all animals in the mixed model were assumed to be genotyped for the QTL.

The effect of using QTL information on the genetic response, the frequency of the favorable QTL allele, and the accuracy of evaluation were examined. Moreover, the effect was studied in four distinct paths of selection: active sires, proven young bulls, bull dams, and firstlactation cows. Average superiority values of 4.6, 7.6, 11.7 , and $1.1 \%$ for genetic response were observed over $16 \mathrm{yr}$ of selection for active sires, young bulls, bull dams, and first-lactation cows, respectively. Frequency of the favorable QTL allele changed faster in bull dams than males, and was the slowest in first-lactation cows. Finally, accuracy of evaluation under the QTL-assisted selection scheme was higher than under the QTL-free selection scheme. Young bulls of the QTL-assisted selection scheme on average had 0.049 higher accuracy, and first-lactation cows had on average 0.185 higher accuracy than corresponding animals of the QTL-free selection scheme.

(Key words: dairy cattle breeding scheme, quantitative trait loci, selection)

Abbreviation key: $\mathbf{A S}=$ active sires, $\mathbf{B D}=$ bull dams, $\mathbf{B V}=$ breeding value, $\mathbf{E B V}$ = estimated breeding value,

Received September 17, 2001.

Accepted January 29, 2002.

Corresponding author: G. Abdel-Azim; e-mail: gaazim@iastate.
$\mathbf{F L C}=$ first lactation cows, $\mathbf{G F D}=$ gene frequency difference, $\mathbf{M A S}=$ marker-assisted selection, $\mathbf{Q A S}=$ QTLassisted selection, $\mathbf{Q F S}=$ QTL-free selection, $\mathbf{Y B}=$ young bulls.

\section{INTRODUCTION}

Classical work in animal breeding has made little use of individual genetic loci in the improvement of quantitative traits in farm animals. The effect that individual loci may have on metric traits and the possibility of selection through known loci were first discussed by Neiman-Sorensen and Robertson (1961) and Smith (1967). However, the recent discovery of several types of DNA markers has allowed for a comprehensive search for associated QTL. Several putative QTL have been found associated with type and production traits in dairy cattle (e.g., Georges et al., 1995; Ashwell et al., 1998; Vukasinovic et al., 1999; Schrooten et al., 2000).

As a result of these recent discoveries, studying the impact that known loci might have on the efficiency of selection becomes essential. The impact of using QTL information in selection has been studied either analytically or by means of computer simulation. Lande and Thompson (1990) analyzed the utilization of markerassisted selection (MAS) in classical schemes of individual and family selection and derived optimal indices combining phenotypic and genomic information to maximize genetic gain. Meuwissen and van Arendonk (1992) used a deterministic simulation model to test the additional genetic gain of MAS within family and found that MAS did not increase rates of genetic gain when applied to conventional progeny testing schemes. Spelman et al. (1999), also using a deterministic simulation model, found limited benefit of MAS in conventional progeny testing schemes. An increase in rate of genetic response of $1.8 \%$ over a base line scheme with no markers was found when $10 \%$ of the genetic variance was associated with the marked QTL. The percent increase became 3.7 with marked QTL variance of $20 \%$. Both Meuwissen and van Arendonk (1992) and Spelman et al. (1999) used a deterministic model to predict the asymptotic genetic gain, ignoring both the reduction in genetic variance from inbreeding and the change in the QTL allele frequency. 
Spelman and Garrick (1998) evaluated two withinfamily MAS schemes using stochastic simulation for a locus that explained $17 \%$ of the genetic variance. A maximum increase of $9 \%$ over a non MAS scheme was found when 40 progeny were simulated for each bull dam. Although Spelman and Garrick (1998) stochastically simulated animals, breeding values were not estimated from data; instead, estimated breeding values were simulated by simulating prediction errors. Also, genetic responses were from a single cycle of MAS, i.e., not from several generations.

Ruane and Colleau (1995) conducted a simulation study of six discrete generations of selection in a closed small population. Single-stage selection was applied to each generation after evaluating animals based on a conventional animal model and a MAS-based animal model (Fernando and Grossman, 1989). Although MAS schemes in general were found to be superior to nonMAS schemes, the study reported inferior MAS polygenic responses to polygenic responses obtained from non-MAS schemes. QTL responses from MAS schemes were, however, distinctively higher than those from non-MAS schemes. The inferior MAS polygenic response was attributed to a reduced accuracy of evaluating the polygenic effects under MAS schemes (Ruane and Colleau, 1995).

Most of the simulation studies found in the literature have used simple breeding structures and small populations with selection for a number of nonoverlapping generations, except with deterministic simulation in which more realistic population structures and overlapping generations were implemented. Also, simulated QTL were rarely estimated by statistical methods but were simulated, except for small populations (e.g., Zhang and Smith, 1993; Meuwissen and Goddard, 1996; Ruane and Colleau, 1995). In other cases, marker or QTL genotypes were directly used in selection in a separate step (e.g., Spelman and Garrick, 1998; Edwards and Page, 1994).

The objective of the current work is to develop a stochastic simulation model that is based on average parameters of the US Holstein population to evaluate the maximum superiority of a QTL-assisted selection (QAS) scheme over a QTL-free selection (QFS) scheme. QAS is based on complete information about a known QTL. Selection is performed for any number of years, $16 \mathrm{yr}$ in the current study, with overlapping generations in a large population. Breeding values and genotype effects are estimated by an animal model.

\section{MATERIALS AND METHODS}

\section{Models for Simulation and Analysis}

Milk yield is evaluated in the United States by an animal model that fits the following components of a cow's lactation record: her management group (m), genetic merit (a), permanent environment, interaction of her herd and sire, parity (p), and a residual component (e) (Wiggans and Vanraden, 1989). In the current simulation, we chose not to simulate or fit permanent environment and sire $\times$ herd interaction factors. The reason was to reduce the complexity of the computational problem. Thus for the ith cow, her phenotypic value of a milk record, say $\mathrm{y}_{\mathrm{i}}$, can be written as

$$
\mathrm{y}_{\mathrm{i}}=\mathrm{m}_{\mathrm{i}}+\mathrm{p}_{\mathrm{i}}+\mathrm{a}_{\mathrm{i}}+\mathrm{e}_{\mathrm{i}}
$$

and based on the simulation model, a general model for analysis can be formulated in matrix form as

$$
\mathbf{y}=\mathbf{X b}+\mathbf{Z a}+\mathbf{e}
$$

where $\mathbf{b}$ is a vector of fixed effects, $\mathbf{a}$ is a vector of random additive genetic effects, and $\mathbf{X}$ and $\mathbf{Z}$ are incidence matrices relating records in $\mathbf{y}$ to fixed and random effects, respectively. Further, $\operatorname{Var}(\mathbf{y})=\sigma_{a}^{2} \mathbf{Z A} \mathbf{Z}^{\prime}+\mathbf{I} \sigma_{e}^{2}$ where $\sigma_{a}^{2} \mathbf{A}$ is the variance covariance matrix of $\mathbf{a}$ with A representing the relationship matrix among animals. The diagonal matrix $\mathbf{I} \sigma_{e}^{2}$ explains the nature of the residual variance which followed from the way they were simulated, i.e., elements of $\mathbf{e},\left\{\mathrm{e}_{\mathrm{i}}\right\}$, were simulated as independent identically distributed $\mathrm{N}\left(0, \sigma_{e}^{2}\right)$. Additive genetic effects, a, were simulated under the assumption of a finite number of loci controlling milk yield. Fixed effects were simulated by adding constants to the record of each individual that corresponded to its levels of fixed factors.

\section{Values of Fixed Effects and Variances of Random Effects}

To simulate a record, appropriate values for the components in equation [1] had to be devised. Kuhn et al. (1994) computed the phenotypic variance of milk records by averaging the within-parity variances reported by Hansen et al. (1983) and adding to it an extra $(249 \mathrm{~kg})^{2}$ due to parity effect. The total phenotypic value was partitioned to $40 \%$ due to fixed factors and $60 \%$ due to random factors (Hansen et al., 1983; Kuhn et al., 1994). Of the $40 \%$, due to fixed components, the herd effect accounted for $22 \%$ and the year effect for $6 \%$. Other fixed factors accounted for the remaining $12 \%$. Similar proportions were used in the current simulation model.

Fixed factors considered in the current simulation were management group and parity. Herd, year, and season subclasses constituted a management group, thus there were four fixed factors whose levels made 
up the columns of $\mathbf{X}$ in [2], a column for each level. Values for herd levels were sampled as in Kuhn et al. (1994) from a normal distribution with mean 0 and variance $0.22(913 \mathrm{~kg})^{2}$, where $(913)^{2}$ is the total phenotypic variance used in the current study. Herd effects were drawn once from the described normal distribution, then they were randomly assigned to base animals. In subsequent years of simulation, a heifer was assigned the same herd effect as her dam.

Six seasons were considered in the current simulation model. Each two consecutive months starting with January constituted the six season levels whose values in kilograms were $179,68,-158,-318,43$, and 187 , respectively. For the parity effect, a value of -249 kilograms was added to first-parity records, and a value of 249 kilograms was added to latter records (Kuhn et al., 1994). A year effect sampled from a normal distribution with mean 0 and variance $0.06(913 \mathrm{~kg})^{2}$ was added to all records generated in this year.

Finally, of the $60 \%$, due to random factors, the additive genetic variance accounted for $\mathrm{h}^{2}(0.6)(913 \mathrm{~kg})^{2}$, and the residual variance accounted for the rest, i.e., (1$\left.\mathrm{h}^{2}\right)(0.6)(913 \mathrm{~kg})^{2}$. In addition, the QTL in the current study explained $15 \%$ of the additive variance, hence the QTL variance was computed as $\mathrm{h}^{2}(0.15)(0.6)(913 \mathrm{~kg})^{2}$, and the polygenic variance was computed as $\mathrm{h}^{2}(0.85)(0.6)(913 \mathrm{~kg})^{2}$.

\section{Simulating Breeding Values}

Breeding value for milk yield was simulated as the aggregate effect of 40 loci in addition to one QTL. The 40 loci, that will be henceforth referred to as the polygenes, were simulated independent of each other and of the QTL. Both the polygenes and the QTL were assumed to have two alleles at each locus. The aggregate polygenic effect was computed as the sum of individual breeding values (BV) associated with each locus, and the total BV of an individual was simulated by adding its aggregate polygenic effect to the BV of the QTL that the individual carries.

Breeding values associated with single loci for base animals. The simulation program requires known proportions for both the polygenic and the QTL additive genetic variances to simulate their corresponding BV. For a single locus of the polygenes, the following steps are taken by the program. First, compute genotypic values in the absence of dominance by taking the square root of $\left(\left(\sigma_{u}^{2} / 40\right) /\left(2 \mathrm{p}_{\mathrm{i}}\left(1-\mathrm{p}_{\mathrm{i}}\right)\right)\right)$ where $\sigma_{u}^{2}$ is the polygenic variance that is divided by 40 to obtain the portion of variance contributed by one locus of the 40 , and $p_{i}$ is the frequency of the favorable allele at the ith locus in the base population. Because the distribution of the $p_{i}$ values is unknown, they were sampled from a uniform distribution with parameters 0 and $1(\operatorname{Unif}(0,1))$. The equal-variance assumption for the polygenes was made to avoid accidental assignment of big variance portions to any of the polygenes in the case of an alternative approach that randomly assigns variance portions to the loci.

The second step is to compute BV according to Falconer (1989) as $2 \mathrm{a}_{\mathrm{i}}\left(1-\mathrm{p}_{\mathrm{i}}\right)$ and $-2 \mathrm{a}_{\mathrm{i}} \mathrm{p}_{\mathrm{i}}$ for homozygotes and $\mathrm{a}_{\mathrm{i}}\left(1-2 \mathrm{p}_{\mathrm{i}}\right)$ for the heterozygote, where $\mathrm{a}_{\mathrm{i}}$ refers to the genotypic value associated with the ith locus of 40 . The same procedures are followed to simulate the BV of the QTL according to the QTL proportion of the genetic variance. As previously mentioned, the QTL was assumed to explain $15 \%$ of the genetic variance, i.e., $49568.588 \mathrm{~kg}$. Given that the frequency of the favorable QTL allele in the base population equals 0.1 , the QTL genotypic value in the absence of dominance equals $353.429 \mathrm{~kg}$, which is 0.39 of the phenotypic standard deviation.

Breeding values for progeny. Genotypes of progeny are constructed from gametes sampled at random from parents' genotypes. A gamete is sampled by drawing with a 0.5 probability one of the two alleles that a parent carries at each locus including the QTL. After forming progeny genotypes, the approach described earlier for simulating BV associated with single loci for base animals was followed to simulate BV for progeny.

\section{Population Structure and Dynamics}

Generally, the simulation addresses an advanced dairy breeding system that utilizes current strategies in selecting dairy sires over two major stages of selection. The first stage includes searching for elite parents to produce young bulls, and the second stage is selecting the best of the progeny tested bulls to become active.

Eighty herds were used in the current study. Herds usually vary in size with frequency that is high for moderate-size herds. This was not considered here since no effect in our model related to herd size. Table 1 summarizes the parameters used in the current simulation.

Cows. An average number of 10,000 milking cows founded the population. The population size was kept constant to avoid genetic gains caused by population growth. To keep the number of milking cows around 10,000 , appropriate culling proportions were used, and a suitable compensatory number of heifers replaced culled cows every year. The culling proportions used for parities 1 to 5 were $0.22,0.26,0.29,0.34$, and 1 , respectively (Kuhn et al., 1994). The number of replacement heifers was determined based on the probability of a 1-yr-old female making a first record. This probability was set so that heifers entering the milking cow popula- 
Table 1. Parameter values used in the simulation program.

\begin{tabular}{l}
\hline Value \\
\hline Genetic \\
Proportion of the genetic variance explained by the QTL: \\
$15 \%$ \\
$\mathrm{~h}^{2}: 0.3$ \\
Initial frequency of the favorable QTL allele: 0.1 \\
Number of alleles at the QTL: 2 \\
Number of QTL: 1 \\
Number of polygenes: 40 with 2 alleles each \\
Nongenetic \\
Number of daughters per young bull (YB): 60 \\
Number of YB: 21 \\
Number of active sires: 10 \\
Number of milking cows: 10,000 \\
Number of progeny per bull dam: 1 \\
Number of herds: 80 \\
Ratios of parities 1 to $5: 0.33,0.26,0.19,0.14,0.09$, respectively. \\
Culling ratios for parities 1 to $5: 0.22,0.26,0.29,0.34$, and 1, \\
respectively.
\end{tabular}

tion almost equals heifers that exit. A third of 10,000 divided by the number of 1-yr-old females was used as the probability of promoting a heifer to a first-parity cow. To further clarify this replacement procedure, let $n_{1}$ denote the number of 1 -yr-old females and $p_{1}$ the probability that a 1-yr-old female makes a record next year, then $p_{1}$ equals $3333 / n_{1}$. Therefore, the expected number of new first-lactation cows $\left(p_{1}\right.$ multiplied by $\left.n_{1}\right)$ equals 3333 females. If $n_{1}<3333, p_{1}$ was set to 1 .

Generating new cow replacements was done by going through the whole list of females and checking age suitability for breeding. If a female was older than $1 \mathrm{yr}$ of age, then her parity was determined; and if a draw from a Unif $(0,1)$ was less than or equal ( 1 - the culling probability given parity), then she stayed. If a female stayed, she produced either a male or a female with equal probabilities. If she produced a male, a new record was generated for her, and she proceeded to the next parity. If she produced a female, she also received a new record and proceeded to the next parity; in addition, her new female offspring was simulated after picking a sire at random from the list of active sires or from the progeny-test bulls. The new female offspring was added to the list of females that the program kept and updated each year.

Bulls. The number of young bulls tested annually was set to 21, and the number of active sires was 10 . Every year, the top $50 \%$ of the active sires are mated to the top cows to produce the 21 young bulls. The process of producing a male is entirely stochastic and similar to what was described about generating female replacements. First, the best female is picked from the milking cow population. Then, based on culling probabilities given parity, the program decides whether she stays or is culled after a draw from a Unif $(0,1)$. If she stays, she is mated to a random sire from the top $50 \%$ of the active sires. But the probability of a male is only $50 \%$, thus a young bull is generated if a draw from a Unif $(0,1)$ does not exceed 0.5 . The next male is generated by repeating the same steps after drawing the next best suitable female.

\section{Selection Criteria}

The simulation program is designed so that selection is always based on an estimated BV (EBV) obtained differently under QAS and QFS. Codes for the simulation and estimation programs may be obtained from Abdel-Azim (2002). Selection is needed first when selecting sires of the future young bulls by selecting the elite $50 \%$ of all active sires based on their EBV. Selection is also needed when the list of active sires is set each year by selecting the top number of active sires out of all males evaluated every year. Males evaluated each year include the active sires of the previous year and the proven young bulls of the current year. Finally, selection is needed when bull dams are chosen by sorting the whole list of milking cows based on cows' EBV, and the best 21 eligible females are selected. The following explains the two selection criteria that were the basis for selection throughout selection practices examined in the current study.

$\boldsymbol{Q T L}$-free selection. This study is intended to compare QTL-assisted selection to current selection practices when implemented in dairy cattle. Current selection practices were simulated in our model as conventional two-stage selection. Active sires for each year were selected based on the animal model, [2], in which elements of the vector a represent the total additive effects of evaluated animals. Each year, after the fifth, proofs of a new set of bulls are computed based on first lactation of their daughters.

QTL-assisted selection. It is assumed that loci affecting milk yield could be physically mapped. Genotypes of loci with large effects can be expressed as independent fixed factors in a mixed linear model, a strategy that provides unbiased estimates of single gene effects when genotypes can be identified for all individuals with observations on the trait (Kennedy et al., 1992). This could be formulated by adding a new factor pertaining to genotypes to model [2] as follows

$$
\mathbf{y}=\mathbf{X b}+\mathbf{X}_{\mathrm{g}} \mathbf{g}+\mathbf{Z u}+\mathbf{e}
$$

where $\mathbf{X}_{\mathrm{g}}$ is an incidence matrix relating genotypes to animals with observations and $\mathbf{g}$ is a vector of genotype effects. Other elements of [3] are defined as in [2], except that $\mathbf{u}$ is a vector of additive genetic effects with the known QTL excluded, i.e., only the polygenic effects. 
The selection criterion investigated here was selecting based on $\mathrm{EBV}_{\mathrm{i}}=\hat{\mathrm{u}}_{\mathrm{ij}}+\hat{\mathrm{g}}_{\mathrm{j}}$ where $\hat{\mathrm{u}}_{\mathrm{ij}}$ and $\hat{\mathrm{g}}_{\mathrm{j}}$ are estimates of the polygenic effect of the ith animal that carries the jth genotype and the QTL effect of the jth genotype, respectively, equation [3]. In the current simulation, all individuals were assumed to have known genotypes for a QTL segregating in the population across families.

\section{Aspects Examined}

Sixteen years of selection were simulated. This is about three cycles of selecting young bulls. In the first $5 \mathrm{yr}$, no analysis was performed because young bulls generated during the first $5 \mathrm{yr}$ were still waiting for their first daughter crop to finish a lactation. In addition, only one set of active sires was simulated at random in the first year and continued to be used throughout the early 5 yr. Hence, there was no genetic improvement in the first $5 \mathrm{yr}$ of selection. In the results section, we do not report selection results of the first $5 \mathrm{yr}$.

For both QAS and QFS, three aspects were addressed: cumulative genetic response, selection pressure, and accuracy of evaluation. These aspects were computed in four distinct groups of animals: active sires (AS), proven young bulls (YB), bull dams (BD), and first-lactation cows (FLC). The advantage of this to designers of breeding programs is to know the pathways of selection that may benefit the most under QAS schemes and also to shed some light on the relationships between trends of genetic response and accuracy of evaluation or selection pressure on the QTL within pathways of selection.

Cumulative genetic response is the most important aspect among the three aspects investigated in the current study. Every year, active sires and bull dams were first selected based on their EBV, and then their true breeding values were averaged. In addition, true breeding values of the proven young bulls and the first-lactation cows were averaged, as well. The average of the true breeding value was used in the current study to indicate the cumulative genetic response. Cumulative genetic responses were presented in terms of percent superiority of specific animals of a selection pathway under the QAS scheme over corresponding animals of the QFS scheme. Mean percent superiority was computed as

$$
2 \sum_{i=1}^{50}\left(Q A S_{i}-Q F S_{i}\right) /\left|Q F S_{i}\right|,\left|Q F S_{i}\right| \neq 0
$$

where $Q A S_{i}$ and $Q F S_{i}$ are the true breeding values averaged for any of the four concerned groups of animals, namely AS, YB, BD, and FLC of the ith replicate of 50. In early years, $Q A S_{i}$ and $Q F S_{i}$ may differ in sign, i.e., negative response for $Q A S_{i}$ and positive for $Q F S_{i}$ or vice versa, although this situation was rarely found in the current study. To avoid computing a negative percent superiority for the QAS scheme when $Q A S_{i}$ is greater than a negative $Q F S_{i}$, division was by the absolute value of $Q F S_{i}$ which is the component $\left|Q F S_{i}\right|$ in formula [4]. Division by the absolute value is also important if $Q F S_{i}$ is greater than a negative $Q A S_{i}$. In addition, the multiplication by 2 in the formula resulted from the multiplication by 100 to convert to percentages and the division by 50 to average over all replicates.

Second, the correlation coefficient between true and EBV was computed separately for AS, YB, BD, and FLC every year and taken to indicate accuracy of EBV under QAS and QFS schemes. Finally, the frequency of the favorable QTL allele was computed within each of the four groups and taken to indicate selection pressure imposed on the QTL in pathways of selection.

The two schemes, QAS and QFS, were replicated 50 times. Results for all replicates were averaged, and standard errors and estimated confidence intervals of the computed means were evaluated, when necessary. In the current simulation, mixed model equations were solved every year using the true variance components to obtain BV estimates. Given that simulation was replicated for 50 times, the equations had to be set and solved 550 times for each scheme each year. In addition, all ancestral and collateral pedigree information were used in a complete relationship matrix inverse which, in turn, produced a large number of equations over the years of simulation. This was a major computational task that limited our resources to only 50 replicates per scheme.

\section{RESULTS AND DISCUSSION}

For the two schemes, QAS and QFS, results are given as an average of 50 replicates. Results include cumulative genetic response, selection pressure on the QTL, and accuracy of evaluation. We first summarize the effect of using QTL information on cumulative genetic response computed for AS, YB, BD, and FLC. Then selection pressure on the QTL in the four pathways is discussed, and finally the accuracy of evaluating YB and FLC is discussed.

\section{Cumulative Genetic Response}

Active sires. On average of all replicates and years, AS of the QAS scheme were $4.6 \%$ higher than AS of the QFS scheme for cumulative genetic response. Table 2 summarizes percent superiority of AS of the QAS scheme over AS of the QFS scheme. Percent superiority started low, then increased and reached a maximum 
Table 2. Percentage of superiority of cumulative genetic response of QTL-assisted selection over QTL-free selection for Active sires.

\begin{tabular}{lllll}
\hline Year & $\begin{array}{l}\text { Superiority } \\
(\%)^{1}\end{array}$ & $\begin{array}{l}\text { Standard } \\
\text { error }\end{array}$ & Minimum $^{2}$ & Maximum $^{2}$ \\
\hline 6 & 1.1 & 0.5 & -5.3 & 14.3 \\
7 & 1.6 & 0.7 & -7.5 & 16.4 \\
8 & 1.9 & 0.8 & -7.4 & 23.4 \\
9 & 2.2 & 0.7 & -7.5 & 12.8 \\
10 & 2.4 & 0.6 & -8.9 & 12.7 \\
11 & 6.3 & 1.6 & -15.8 & 49.3 \\
12 & 7.1 & 1.5 & -10.8 & 32.0 \\
13 & 7.0 & 1.6 & -12.9 & 35.7 \\
14 & 7.3 & 1.5 & -11.7 & 24.8 \\
15 & 7.2 & 1.2 & -11.1 & 34.4 \\
16 & 6.0 & 1.4 & -15.9 & 49.5 \\
Overall $^{3}$ & 4.6 & 0.4 & -15.9 & \\
\hline
\end{tabular}

${ }^{1}$ Average of 50 replicates within a year.

${ }^{2}$ Minimum or maximum of 50 replicates within a year.

${ }^{3}$ Computed for all replicates and years.

in the third cycle of selection, and finally started to decrease again at the end of the third cycle of selection.

This superiority of AS of the QAS scheme varied from a minimum of $1.1 \%$ in yr 6 to a maximum of $7.3 \%$ in yr 14. In addition, large variation was noticed across the 50 replicates: The worst group of AS was observed in yr 16 for one of the replicates, and the best group was observed at yr 12 for another replicate. The worst group was $15.9 \%$ inferior and the best group was $49.5 \%$ superior to AS of the QFS scheme.

Young bulls. Only results of yr 10 to 16 are shown in Table 3 for the proven YB for the following reason. The YB of both QAS and QFS schemes were exactly the same until yr 9. Proven YB of yr 6, 7, 8, and 9 were born in yr 1,2,3, and 4, respectively, and were sampled for progeny testing at one year of age in yr 2, 3, 4, and 5 , respectively. Estimated BV were not available for animals before yr 6, and selection of parents of young bulls was at random. Because seed numbers used for random number generators were the same for the QAS and QFS schemes, the same set of parents and the resulting young bulls were sampled for both schemes until yr 5 .

On average of all replicates for yr 10 to 16, proven YB of the QAS scheme were $7.6 \%$ higher than proven YB of the QFS scheme, Table 3. The superiority of YB of the QAS scheme ranged from a minimum of $3.8 \%$ at yr 10 to a maximum of $10.5 \%$ at yr 14 . This is similar to the trend observed for AS. The similarity being a low early superiority that reached a maximum in the third cycle of selection and then dropped. However, percent superiority of proven YB was better than AS, which was caused by the less progeny information available for YB than AS. On average, YB had 60 daughters each, and active sires had 400 daughters each. In reality, the number of daughters of AS may exceed 400, which should further enlarge the difference in percent superiority between AS and YB of the QAS scheme.

The variation across the 50 replicates was greater than the corresponding variation in AS. YB are ex-

Table 3. Percentage of superiority of cumulative genetic response of QTL-assisted selection over QTL-free selection for young bulls.

\begin{tabular}{lllll}
\hline Year & $\begin{array}{l}\text { Superiority } \\
(\%)^{1}\end{array}$ & $\begin{array}{l}\text { Standard } \\
\text { error }\end{array}$ & Minimum $^{2}$ & Maximum $^{2}$ \\
\hline 10 & 3.8 & 1.9 & -24.5 & 45.4 \\
11 & 8.0 & 2.2 & -17.3 & 42.0 \\
12 & 4.2 & 2.5 & -26.7 & 80.6 \\
13 & 10.0 & 1.8 & -10.9 & 41.3 \\
14 & 10.5 & 1.7 & -13.5 & 44.3 \\
15 & 7.7 & 2.0 & -18.3 & 40.9 \\
16 & 9.0 & 1.6 & -10.8 & 80.6 \\
Overall $^{3}$ & 7.6 & 0.7 & -26.7 & \\
\hline
\end{tabular}

${ }^{1}$ Average of 50 replicates within a year.

${ }^{2}$ Minimum or maximum of 50 replicates within a year.

${ }^{3}$ Computed for all replicates and years. 
Table 4. Percentage of superiority of cumulative genetic response of QTL-assisted selection over QTL-free selection for bull dams.

\begin{tabular}{lclcc}
\hline Year & $\begin{array}{l}\text { Superiority } \\
(\%)^{1}\end{array}$ & $\begin{array}{l}\text { Standard } \\
\text { error }\end{array}$ & Minimum $^{2}$ & Maximum $^{2}$ \\
\hline 6 & 8.9 & 1.3 & -5.6 & 36.6 \\
7 & 9.5 & 1.4 & -11.8 & 36.9 \\
8 & 8.8 & 1.6 & -14.8 & 29.4 \\
9 & 13.6 & 1.6 & -8.1 & 48.5 \\
10 & 12.9 & 1.4 & -12.0 & 41.6 \\
11 & 12.1 & 1.4 & -10.5 & 38.8 \\
12 & 11.4 & 1.4 & -7.0 & 33.7 \\
13 & 14.1 & 1.4 & -10.6 & 38.2 \\
14 & 13.6 & 1.4 & -7.2 & 34.0 \\
15 & 14.5 & 1.4 & -3.8 & 42.9 \\
16 & 9.2 & 1.4 & -8.8 & 48.5 \\
Overall $^{3}$ & 11.7 & 0.4 & -14.8 & \\
\hline
\end{tabular}

${ }^{1}$ Average of 50 replicates within a year.

${ }^{2}$ Minimum or maximum of 50 replicates within a year.

${ }^{3}$ Computed for all replicates and years.

pected to have more variation than AS due to their larger number (or equivalently their smaller selection intensity) and their less accuracy of evaluation than AS. The range of variation extended from the worst group of proven YB under the QAS scheme that had an inferiority of $26.7 \%$ to the best group that had a superiority of $80.6 \%$ relative to corresponding animals of the QFS scheme.

Bull dams. Percent superiority of $\mathrm{BD}$ of the QAS scheme over BD of the QFS scheme is presented in Table 4. Percent superiority in $\mathrm{BD}$ was the highest among the four groups of animals studied. On average of all replicates and years, BD of the QAS scheme was $11.7 \%$ higher than $\mathrm{BD}$ of the QFS scheme. The superiority ranged from a minimum of $8.8 \%$ at yr 8 to a maximum of 14.5 at yr 15 . The trend of starting low superiority that increased to a maximum during the last cycle of selection and then dropped was similar to what was found for AS and YB.

The range of variation was similar to the range of variation found in YB and similar to the range of variation found in AS. The lowest group of BD was 14.8\% inferior and the highest group was $48.5 \%$ superior in QAS over QFS scheme (Table 4). One reason for the smaller range of variation observed in $\mathrm{BD}$ than $\mathrm{YB}$ was the higher selection intensity of BD than YB.

First-lactation cows. Cows in general benefited the least from using QTL information partly because not enough time existed for parental superiority to be expressed in the cows. On average, of all replicates and years, superiority of $1.1 \%$ was found for the FLC of the QAS scheme over FLC of the QFS scheme. However, a high standard error of 1.1 for the superiority average, $1.1 \%$, was also found. As a result, the mean value of superiority of response in FLC was not significantly different from 0 . The $95 \%$ confidence interval for the mean was $(-1.002,3.180)$. Table 5 shows the frequency distribution of percent superiority for all datasets. The frequency distribution shows a greater number of positive percent superiority than negative. Table 5, however, shows two outlier values: $-500 \%$ and $143 \%$. Outliers were common in FLC in yr 6 to 9 of selection because of sign differences between genetic responses obtained from QAS and QFS schemes. For example, the $-500 \%$ value resulted from an average $\mathrm{BV}$ of $-1.814 \mathrm{~kg}$ for the QAS scheme and $0.454 \mathrm{~kg}$ for the QFS scheme in yr 7. The two values are potentially 0 and could better be excluded. Generally for FLC, yr 6 to 9 were not a good indication of genetic response because their parents were randomly generated or chosen before yr 6 .

Table 6 lists FLC percent superiorities of the QAS over the QFS scheme for yr 10 to 16 only. The overall mean for these years, as the table shows, is higher than the mean for yr 6 to 16 . The superiority mean as shown is $2.5 \%$, with a standard error of 0.4 and a $95 \%$ confidence interval of $(1.699,3.295)$. Percent superiority of QAS shows an only-increasing trend for FLC, from -0.6

Table 5. Frequency distribution of percentage of superiority of QTLassisted selection over QTL-free selection for first-lactation cows.

\begin{tabular}{lcc}
\hline From $(\geq)$ & To $(<)$ & Count \\
\hline-500 & -429 & 1 \\
-429 & -357 & 0 \\
-357 & -286 & 0 \\
-286 & -214 & 0 \\
-214 & -143 & 0 \\
-143 & -71 & 0 \\
-71 & 0 & 209 \\
0 & 71 & 339 \\
71 & 143 & 0 \\
143 & 214 & 1 \\
\hline
\end{tabular}


Table 6. Percentage of superiority of cumulative genetic response of QTL-assisted selection over QTL-free selection for first-lactation cows.

\begin{tabular}{lllll}
\hline Year & $\begin{array}{l}\text { Superiority } \\
(\%)^{1}\end{array}$ & $\begin{array}{l}\text { Standard } \\
\text { error }\end{array}$ & Minimum $^{2}$ & Maximum $^{2}$ \\
\hline 10 & -0.6 & 1.0 & -18.0 & 11.0 \\
11 & 0.3 & 0.9 & -13.0 & 18.8 \\
12 & 1.4 & 0.7 & -19.5 & 13.7 \\
13 & 3.6 & 1.1 & -14.7 & 17.0 \\
14 & 4.0 & 1.1 & -9.5 & 33.6 \\
15 & 4.0 & 1.3 & -7.6 & 26.6 \\
16 & 4.8 & 1.2 & -9.0 & 35.1 \\
Overall $^{3}$ & 2.5 & 0.4 & -19.5 & \\
\hline
\end{tabular}

\footnotetext{
${ }^{1}$ Average of 50 replicates within a year.

${ }^{2}$ Minimum or maximum of 50 replicates within a year.

${ }^{3}$ Computed for all replicates and years.
}

to 4.8 (Table 6). However, according to other paths of selection previously discussed, the trend was increasing for a limited amount of time, then it ceased and decreased. This only-increasing trend in FLC is a result of the genetic response lag of the cows behind AS and $\mathrm{BD}$, i.e., the extra genetic superiority initially observed in AS and BD ultimately will be expressed in FLC.

Generally, all paths of selection benefited from using a QTL that explained 15\% of the additive variance. Actual cumulative response values were not given, instead superiority of QAS over QFS as a percentage was given. For the actual cumulative genetic response values, refer to Table 7. Ruane and Colleau (1996), who also used stochastic simulation and estimated genetic effects by realistic statistical procedures, found an overall superiority of $6.8 \%$ in the genetic response with a marked QTL that explained $12.5 \%$ of the additive variance. The $6.8 \%$ was an overall average of all animals in the breeding program when heritability was 0.25 and the initial frequency of the favorable QTL allele was 0.1 . This superiority is similar or higher than what was found in the current study. However, Ruane and Colleau (1996) simulated a closed nucleus herd in which bulls were selected once per generation through a single stage of selection. Although bulls were evaluated based on 64 daughters, the daughters were composed of two full sib families of 32 each. For the next generation (generations were discrete) one female was selected from each full sib family, and one male was selected from each two full sib families. This situation is highly advantageous for a MAS scheme, which excels in exploiting the within-family variation particularly with the highly polymorphic marker used by Ruane and Colleau (1996); $2 \mathrm{~N}$ different marker alleles were generated for the $\mathrm{N}$ foundation animals.

Genetic response for bull dams was the highest among all pathways. Spelman et al. (1999) found similar advantage for the bull dams pathway; of the increase in genetic gain 80 to $95 \%$ was from the cow to bull path.

\section{Selection Pressure on the QTL}

Measuring frequency of QTL alleles over years of selection is a possibility that stochastic simulation offers. This section discusses the change in frequency of the favorable QTL allele for the four paths of selection. In the current study, the change in gene frequency is taken to express selection pressure on the favorable QTL allele. Gene frequency is expected to be higher for animals of the QAS scheme over animals of the QFS scheme. Gene frequency of animals of the QAS scheme minus gene frequency of animals of the QFS scheme will be henceforth referred to as gene frequency difference (GFD). GFD indicates the superiority of the QAS over the QFS scheme in pushing the favorable QTL allele towards fixation.

Table 7. Average of cumulative genetic response for QTL-assisted selection (QAS) and QTL-free selection (QFS) schemes in kilograms of milk.

\begin{tabular}{|c|c|c|c|c|c|c|c|c|}
\hline \multirow[b]{2}{*}{ Period } & \multicolumn{2}{|c|}{ Active sires } & \multicolumn{2}{|c|}{ Young bulls } & \multicolumn{2}{|c|}{ Bull dams } & \multicolumn{2}{|c|}{$\begin{array}{c}\text { First-lactation } \\
\text { cows }\end{array}$} \\
\hline & QAS & QFS & QAS & QFS & QAS & QFS & QAS & QFS \\
\hline All yr & 924 & 881 & 576 & 539 & 1172 & 1054 & 430 & 420 \\
\hline $6-10 \mathrm{yr}$ & 543 & 534 & 139 & 135 & 948 & 858 & 152 & 153 \\
\hline $11-16$ yr & 1242 & 1171 & 939 & 876 & 1359 & 1217 & 661 & 643 \\
\hline
\end{tabular}


Table 8. Frequency of the favorable QTL allele in males under QTL-assisted selection (QAS) and QTL-free selection (QFS) schemes.

\begin{tabular}{|c|c|c|c|c|c|c|}
\hline \multirow[b]{2}{*}{ Year } & \multicolumn{2}{|c|}{ Frequency } & \multicolumn{2}{|c|}{ Minimum $^{1}$} & \multicolumn{2}{|c|}{ Maximum $^{1}$} \\
\hline & QAS & QFS & QAS & QFS & QAS & QFS \\
\hline 6 & 0.127 & 0.125 & 0.016 & 0.016 & 0.306 & 0.306 \\
\hline 7 & 0.145 & 0.145 & 0.0 & 0.0 & 0.274 & 0.290 \\
\hline 8 & 0.159 & 0.154 & 0.016 & 0.016 & 0.323 & 0.323 \\
\hline 9 & 0.163 & 0.160 & 0.016 & 0.0 & 0.355 & 0.339 \\
\hline 10 & 0.399 & 0.309 & 0.129 & 0.032 & 0.726 & 0.774 \\
\hline 11 & 0.501 & 0.369 & 0.242 & 0.032 & 0.742 & 0.758 \\
\hline 12 & 0.573 & 0.417 & 0.161 & 0.048 & 0.871 & 0.832 \\
\hline 13 & 0.621 & 0.422 & 0.274 & 0.129 & 0.871 & 0.774 \\
\hline 14 & 0.645 & 0.462 & 0.323 & 0.097 & 0.903 & 0.823 \\
\hline 15 & 0.775 & 0.543 & 0.419 & 0.129 & 0.952 & 0.952 \\
\hline 16 & 0.825 & 0.588 & 0.468 & 0.226 & 1.0 & 0.935 \\
\hline
\end{tabular}

${ }^{1}$ Minimum or maximum of 50 replicates within a year.

Males. Table 8 shows the annual change in gene frequency as an average of all replicates for males (active sires and young bulls). Gene frequency increased from 0.127 to 0.825 in $10 \mathrm{yr}$ of QAS and increased from 0.125 to 0.588 in $10 \mathrm{yr}$ of QFS. Maximum values show that the favorable allele was even fixed in some replicates under QAS and reached close-to-fixation values under QFS. This indicates the short time horizon needed to fix a QTL of the size used in the current simulation, which, in turn, justifies designing breeding programs to benefit from QTL information only in the short run. The results also cast a great deal of doubt about the possibility of finding a QTL of size 0.39 phenotypic standard deviation at a low frequency of the favorable allele in similarly selected populations. Future artificial intervention and mutations are still possible as ways of introducing new genes to such selected populations.

Although both QAS and QFS schemes raised the favorable QTL allele frequency, the QAS scheme surpassed the QFS scheme with consistently positive GFD that increased at a high rate early in selection and slower rate near the end. Figure 1 is a plot of the GFD for males, BD, and FLC.

Bull dams. Table 9 lists favorable QTL allele frequency in bull dams averaged over 50 replicates. Minimum and maximum values observed across the 50 replicates for each year are also shown. The table shows that the gene frequency in bull dams in year 6 was 0.675 , with a maximum of 1 under the QAS scheme. Also, a $15.8 \%$ probability that gene frequency in bull dams exceeds 0.995 was estimated from data in yr 6 . This high starting frequency of the favorable QTL allele in BD of the QAS scheme clearly shows the high selection pressure imposed on the favorable QTL allele in $B D$ very early in selection. These results agree with the starting $8.9 \%$ superiority in genetic response of BD of the QAS scheme shown in Table 4.
The trend of GFD in BD was similar to males. An increasing rate at early stages of selection followed by a decreasing rate. However, unlike males, the trend of GFD in BD decreased after that, as shown in Figure 1. This is very likely due to the significantly higher selection pressure on the QTL in BD than in males. A similar decrease in GFD is also expected to appear in males for a longer time horizon when the favorable QTL allele for QAS reaches a frequency that is as high as in BD. Notice that in yr 13 under QAS, the frequency average of the favorable QTL allele in BD was 0.947 (Table 9). This reversed the increasing trend of the GFD because of the very limited distance left to reach the ceiling frequency of 1 . On the other hand, the corresponding frequency in males under QAS was still 0.621 (Table 8), allowing for GFD to continue to increase.

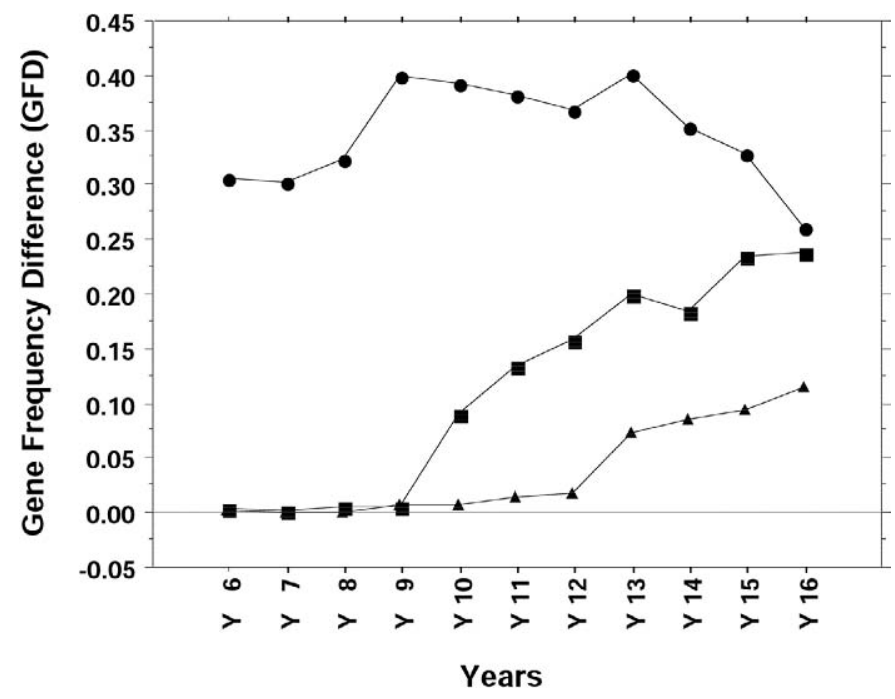

Figure 1. Gene frequency difference (frequency of animals of QAS scheme minus corresponding animals of QFS scheme) computed for males (ם), bull dams $(\bullet)$, and first-lactation cows $(\mathbf{\Lambda})$. 
Table 9. Frequency of the favorable QTL allele in Bull dams under QTL-assisted selection (QAS) and QTLfree selection (QFS) schemes.

\begin{tabular}{|c|c|c|c|c|c|c|}
\hline \multirow[b]{2}{*}{ Year } & \multicolumn{2}{|c|}{ Frequency } & \multicolumn{2}{|c|}{ Minimum $^{1}$} & \multicolumn{2}{|c|}{ Maximum $^{1}$} \\
\hline & QAS & QFS & QAS & QFS & QAS & QFS \\
\hline 6 & 0.675 & 0.370 & 0.381 & 0.119 & 1.0 & 0.667 \\
\hline 7 & 0.675 & 0.375 & 0.381 & 0.095 & 0.929 & 0.643 \\
\hline 8 & 0.717 & 0.395 & 0.310 & 0.143 & 0.976 & 0.690 \\
\hline 9 & 0.796 & 0.397 & 0.476 & 0.095 & 1.0 & 0.786 \\
\hline 10 & 0.835 & 0.444 & 0.500 & 0.143 & 1.0 & 0.738 \\
\hline 11 & 0.860 & 0.480 & 0.595 & 0.095 & 1.0 & 0.833 \\
\hline 12 & 0.887 & 0.520 & 0.643 & 0.167 & 1.0 & 0.857 \\
\hline 13 & 0.947 & 0.547 & 0.690 & 0.167 & 1.0 & 0.929 \\
\hline 14 & 0.959 & 0.608 & 0.810 & 0.143 & 1.0 & 0.929 \\
\hline 15 & 0.985 & 0.658 & 0.929 & 0.262 & 1.0 & 0.976 \\
\hline 16 & 0.986 & 0.727 & 0.905 & 0.286 & 1.0 & 0.952 \\
\hline
\end{tabular}

${ }^{1}$ Minimum or maximum of 50 replicates within a year.

First-lactation cows. Similar to genetic superiority, differences in GFD between paths are the result of genetic lag and gene flow. Frequency average of the favorable QTL allele for FLC started at 0.1 for QAS and QFS in yr 6 and increased to 0.566 and 0.453 for QAS and QFS, respectively, in yr 16. In FLC, GFD was the least among other paths but continued to increase (Figure 1).

\section{Accuracy of Evaluation}

This section uses the correlation coefficient between true and estimated BV of proven $\mathrm{YB}$ to express accuracy of evaluation under the two schemes studied. Figure 2 displays accuracy for all years for both QAS and QFS schemes. Proven YB of QAS were consistently superior to corresponding animals under the QFS scheme. The

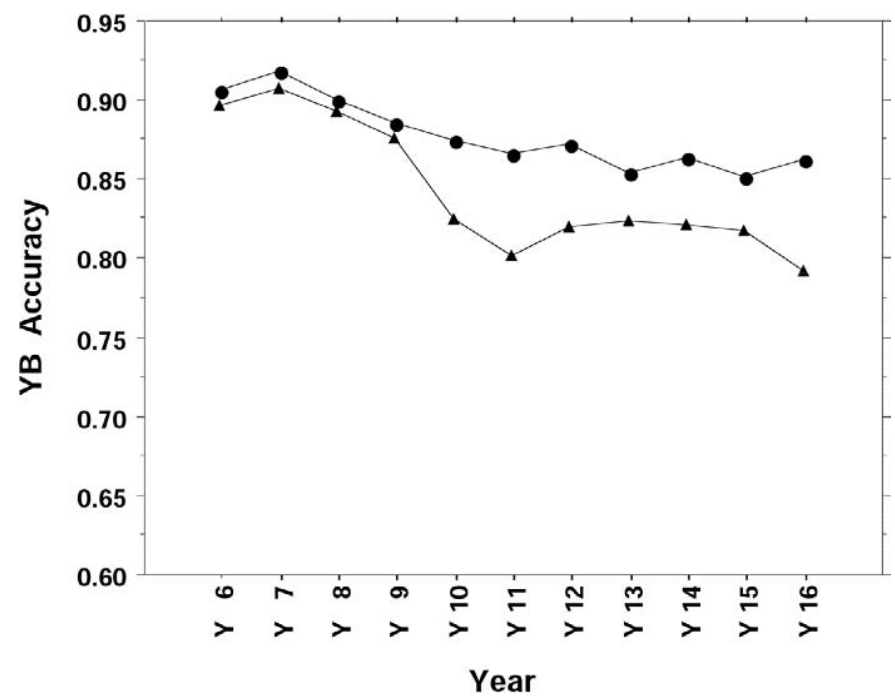

Figure 2. Accuracy of estimating breeding values for young bulls under QTL-assisted selection $(\bullet)$ and QTL-free selection $(\boldsymbol{\Delta})$. difference between QAS and QFS was averaged and found to be 0.035 for yr 6 to 16 and 0.049 for yr 11 to 16 , i.e., the last cycle of selection. These results indicate that under QAS, YB in the second selection stage are more accurately evaluated. This means less risk involved with promoting young bulls to active sires. Notice, however, that the difference in accuracy was small.

Accuracy in other paths of selection was not addressed for the following reasons. First, for AS, they were the path with the most amount of information in terms of progeny information, hence their accuracy of evaluation is often too high for QTL information to enhance. In other words, in practice animal breeders are more worried about the accuracy of evaluating their YB than AS that have thousands of daughters and hence are evaluated with a maximum possible accuracy. Second, for $\mathrm{BD}$, their age distribution was not the same in QAS and QFS schemes. In fact, age average of $\mathrm{BD}$ under QAS scheme was less than their age average under QFS scheme (Table 10). Age distribution difference makes comparing accuracy of evaluating $\mathrm{BD}$ under QAS and QFS schemes less meaningful. Also, the correlation between true and estimated BV for bull dams is severely biased by selection.

Finally for the FLC, they are not produced by selecting them but by selecting their parents, and hence the correlation between their estimated and true $\mathrm{BV}$ is not specifically important to future FLC. However, under QAS, a greater proportion of BD is likely to come from FLC as Table 10 indicates for the current simulation. Therefore, a high accuracy of evaluating FLC is essential for BD selection. Figure 3 is a plot of the accuracy of evaluation in FLC. The graph clearly shows an increasing superiority of FLC of the QAS scheme over FLC of the QFS scheme. The superiority is shown to be greater than the corresponding superiority of the proven YB (Figure 2). The average superiority in accu- 
Table 10. Average number of lactations and their proportions for bull dams for QTL-assisted selection (QAS) and QTL-free selection (QFS).

\begin{tabular}{llcc}
\hline \multirow{2}{*}{ Average number of lactations } & \multicolumn{2}{c}{ Average over all replicates } \\
\cline { 3 - 4 } & & QAS & QFS \\
\hline \multirow{3}{*}{ Proportion } & & 2.19 & 2.82 \\
of & First & 0.36 & 0.11 \\
lactation & Second & 0.30 & 0.35 \\
& Third & 0.17 & 0.26 \\
& Fourth & 0.11 & 0.19 \\
& Fifth & 0.05 & 0.10 \\
\hline
\end{tabular}

racy of QAS scheme over QFS scheme for FLC was 0.151 for yr 6 to 16 and 0.185 for yr 11 to 16 . The corresponding superiority values in proven YB were 0.035 and 0.049 . The better accuracy superiority observed for FLC was because of the less progeny information available for cows than YB.

\section{CONCLUSIONS}

This study showed a consistent advantage of QAS over QFS in all pathways of selection in conventional breeding schemes of dairy cattle. The rate of increase of the superiority of QAS over QFS in highly selected pathways such as in BD started very high then slowed down and finally decreased before yr 16 . This decrease in superiority was accompanied by a high QTL allele frequency, i.e., as the QTL reached fixation, the advantage of using it in genetic evaluation decreased. Similar trends are expected in all pathways of selection for longer time horizon.

For designing breeding programs that consider QTL information, attention must be paid to improvement of

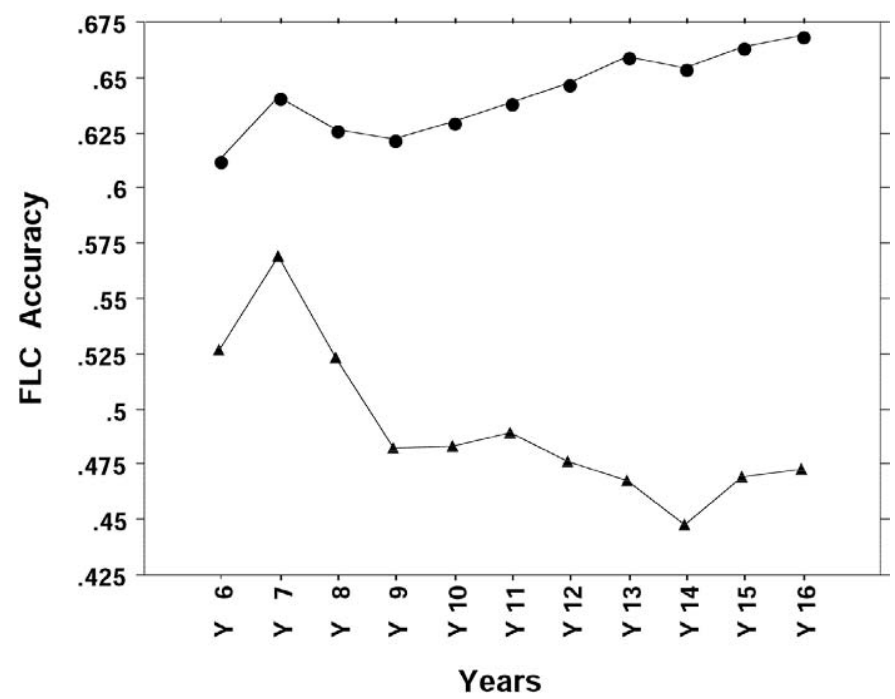

Figure 3. Accuracy of estimating breeding values for first-lactation cows under QTL-assisted selection $(\bullet)$ and QTL-free selection $(\mathbf{\Delta})$. distinct pathways that benefit the most from using QTL information which eventually benefits the whole population. As we showed, the advantage of QAS increases significantly and decreases at different times in the future for the four pathways of selection. Future work to test breeding programs that shorten the 5-year waiting period of young bulls and optimum schemes that employ nucleus herds with multiple ovulation and embryo transfer (MOET) is in progress.

The simulation was stochastic and allowed for studying the frequency of the favorable QTL allele by directly computing the favorable allele every year from data for the different pathways of selection. QTL allele frequency reached close-to-fixation values in $\mathrm{BD}$ in a short time. Also, it increased significantly in other pathways of selection. Therefore, using information about QTL equal or greater than 0.39 phenotypic standard deviation units may be beneficial only in the short run. However, the high rate of increase of the frequency of the favorable QTL allele under schemes of QFS found in the current study casts a great deal of doubt about the possibility of finding QTL or marked QTL of the size presented here, 0.39 phenotypic standard deviation units, at low frequency. Gibson (1994) showed that the favorable QTL allele reached fixation under mass selection that gave better long-term response when genotype information was ignored. Future artificial intervention and mutations as ways of introducing new genes to selected populations at low frequency are still possible.

\section{REFERENCES}

Abdel-Azim, G. 2002. http://www.public.iastate.edu/ gaazim/simulate/mas_overview.html. Accessed Jan. 9, 2002.

Ashwell, M. S., Y. Da, C. P. Van Tassell, P. M. Vanraden, R. H. Miller, and C. E. Rexroad, Jr. 1998. Detection of putative trait loci affecting milk production and composition, health, and type traits in a United States Holstein population. J. Dairy Sci. 81:3309-3314.

Edwards, M. D., and N. J. Page. 1994. Evaluation of marker-assisted selection through computer simulation. Theor. Appl. Genet. 88:376-382.

Falconer, D. S. 1989. Introduction to Quantitative Genetics. 3rd ed. Longman, New York.

Fernando, R. L., and M. Grossman. 1989. Marker assisted selection using best linear unbiased prediction. Genet. Sel. Evol. 21:467-477. 
Georges, M., D. Nielsen, M. Mackinnon, A. Mishra, R. Okimoto, A. T. Pasquino, L. S. Sargeant, A. Sorensen, M. R. Steele, X. Zhao, J. E. Womack, and I. Hoeschele. 1995. Mapping quantitative trait loci controlling milk production in dairy cattle by exploring progeny testing. Genetics 139:907-920.

Gibson, J. P. 1994. Short-term gain at the expense of long-term response with selection of identified loci. Proc. 5th World Congr. Genet. Appl. Livest. Prod., Guelph, Canada 21:202-204.

Hansen, L. B., A. E. Freeman, and P. J. Berger. 1983. Variances, repeatabilities, and age adjustments of yield and fertility in dairy cattle. J. Dairy Sci. 66:281-292.

Kennedy, B. W., M. Quinton, and J. A. M. van Arendonk. 1992. Estimation of effects of single genes on quantitative traits. J. Anim. Sci. 70:2000-2012.

Kuhn, M. T., P. J. Boettcher, and A. E. Freeman. 1994. Potential biases in predicted transmitting abilities of females from preferential treatment. J. Dairy Sci. 77:2428-2437.

Lande, R., and R. Thompson. 1990. Efficiency of marker-assisted selection in the improvement of quantitative traits. Genetics 124:743-756.

Meuwissen, T. H. E., and J. A. M. van Arendonk. 1992. Potential improvement in rate of genetic gain from marker-assisted selection in dairy cattle breeding schemes. J. Dairy Sci. 75:1651-1659.

Meuwissen, T. H. E., and M. E. Goddard. 1996. The use of marker haplotypes in animal breeding schemes. Genet. Sel. Evol. 28:161-176.

Neiman-Sorensen, A., and A. Robertson. 1961. The association between blood groups and several production characters in three Danish cattle breeds. Acta Agric. Scand. 11:163-196.
Ruane, J., and J. J. Colleau. 1995. Marker-assisted selection for genetic improvement of animal populations when a single QTL is marked. Genet. Res. 66:71-83.

Ruane, J., and J. J. Colleau. 1996. Marker-assisted selection for a sex limited character in a nucleus breeding population. J Dairy Sci. 79:1666-1678.

Schrooten, C., H. Bovenhuis, W. Coppieters, and J. A. M. van Arendonk. 2000. Whole genome scan to detect quantitative trait loci for conformation and functional traits in dairy cattle. J. Dairy Sci. 83:795-806.

Smith, C. 1967. Improvement of metric traits through specific genetic loci. Anim. Prod. 9:349-358.

Spelman, R. J., and D. J. Garrick. 1998. Genetic and economic responses for within-family marker-assisted selection in dairy cattle breeding schemes. J. Dairy Sci. 81:2942-2950.

Spelman, R. J., D. J. Garrick, and J. A. M. van Arendonk. 1999. Utilisation of genetic variation by marker assisted selection in commercial dairy cattle populations. Livest. Prod. Sci. 59:51-60.

Vukasinovic, N., S. K. Denise, and A. E. Freeman. 1999. Association of growth hormone loci with milk yield traits in Holstein bulls. J. Dairy Sci. 82:788-794.

Wiggans, G. R. and P. M. Vanraden. 1989. USDA-DHIA Animal model genetic evaluations. http://www.inform.umd.edu/EdRes/Topic/ AgrEnv/ndd/dairy/ANIMAL_MOD EL_GENETIC_EVALUATIONS.html. Accessed: Feb. 2, 2001.

Zhang W., and C. Smith. 1993. Simulation of marker assisted selection utilizing linkage disequilibrium: the effects of several additional factors. Theor. Appl. Genet. 86:491-496. 